\title{
Exact and approximation algorithms for joint routing and flow rate optimization
}

\author{
Konstanty Junosza-Szaniawski \\ Warsaw University of Technology \\ Faculty of Mathematics and Information Science \\ ul. Koszykowa 75, 00-662 Warszawa, Poland \\ Email: k.szaniawski@mini.pw.edu.pl
}

\author{
Dariusz Nogalski \\ Military Communication Institute \\ C4I Systems Department \\ ul. Warszawska 22A, 05-130 Zegrze, Poland \\ Email: d.nogalski@wil.waw.pl
}

\begin{abstract}
This paper addresses the comparison of algorithms for a version of the Network Utility Maximization (NUM) problem. The joint formulation of routing and transmission rate control within the multi-user and single-path setting is assumed within the NUM. Since the problem is NP-hard, the efficient heuristics are designed, implemented and compared experimentally with other existing heuristics and exact linear programming solver. The linear approximation is applied for a nonlinear utility function. The results of the experiments demonstrate a trade-off between computing time and precision of goal value.
\end{abstract}

Index Terms-Mathematical programming, network optimization problem, network utility maximization, rate allocation, congestion control, multi-rate computer networks, routing, NPhard problem, heuristic algorithm

\section{INTRODUCTION}

The Network Utility Maximization (NUM) problem was introduced by Kelly et al. in 1998 [10]. Within the problem a single route is associated with a user (constant to the problem) and the goal is to maximize aggregate utility of all user rates (variable to the problem). Such a basic NUM problem is known to be polynomial-time solvable [17]. We refer to such a problem as the basic NUM.

This article addresses an extended problem where both user rates and routes are variable to the problem. We refer to such a problem as the extended NUM problem or simply, the $N U M$ problem. Wang et al. prove such a problem to be NP-hard [17]. As a consequence, efficient heuristics are needed for larger networks.

Such a joint formulation of the problem is useful since path selection determines link congestion and the problem can be solved efficiently. Efficiency is becoming a critical problem in many application areas, e.g. in multimedia WSN (Wireless Sensor Networks). Multimedia data producing wireless sensor nodes require higher bandwidth, higher energy usage, and strict quality of service (QoS) requirements [13]. Additionally, we are interested in techniques to achieve fairness of multistream allocations [10] [11] [3].

We designed and implemented several heuristics and compared their performance with other existing heuristics and the exact linear programming solver. Due to the fact that our utility function was nonlinear, we applied linear approximation within optimization procedure. The results of the experiments demonstrate a trade-off between computing time and precision of algorithms.

Since our approach is centralized, it cannot be used directly in multi-rate networks operating in a distributed way. However, there are certain applications where our heuristics can be found useful. Firstly, in applications where centralized network controllers are applied, e.g. SDN. Secondly, to estimate a gap between single and multiple path solutions [18].

\section{Motivation AND RELATED WORK}

A. The complexity of various versions of network utility maximization problem

The complexity of the basic and the extended NUM was stated in the introduction. However, for various problem modifications it may differ. The decision version of $m$-commodity flow problem with fixed rates and single-path setting is NPcomplete [4]. It is proved by reduction from the decision version of the bin-packing problem. In [5] it is shown that even the decision version of two-commodity integral flow problem is NP-complete, by reduction from the SAT problem.

\section{B. Distributed models}

Since the work of Kelly et al. [10] was published, there have been many works on price-based distributed network utility maximizing methods. Chiang et al. [3] present the TCP/IP joint optimization example and solve it through distributed decomposition. Horizontal decomposition of TCP-AQM Congestion Control to share link capacities among competing users is described. Vertical decomposition of TCP/IP Joint Congestion Control and Routing is described.

\section{Centralized control in SDN networks}

Amokrane el al. [1] propose an online energy efficient flowbased routing approach for SDN controller, which allows for dynamic reconfiguration of existing flows (paths and rates). Huang et al. [8] solve utility-optimized flow-level bandwidth allocation in hybrid SDNs.

\section{Importance of single-path routing}

Single-path routing is still important from the practical point of view in the following areas: hybrid SDN networks [8], energy-aware networks [1] [9], IP networks [3] [10], 
MANETs (Mobile Ad hoc NETworks) [16] and WSNs [13]. It imposes a smaller overhead in implementation and is less expensive to support, as opposed to the multi-path [18]. Multipath routing, as opposed to single-path routing has several disadvantages/challenges: data packet reordering, route maintenance, route discovery and a computational overhead.

\section{E. Fairness and Efficiency}

In literature there are various definitions of fairness. Two of them are most common: Max-min fairness [14], and proportional fairness [10] [14] [19] [11] [3]. The proportional fair vector comes as a solution to maximization of aggregate utility given by the equation (11) . Utility functions can be interpreted as the level of elasticity of application traffic, user satisfaction, optimality of resource allocation efficiency, as well as fairness [3]. If $\alpha=0$, the NUM problem reduces to system throughput maximization. If $\alpha=1$, proportional fairness among competing users is attained. And finally, if $\alpha=\infty$, then max-min fairness is achieved (Lee et al. [11]). The improvement in fairness is achieved by sacrificing the network efficiency. As the value of $\alpha$ increases, the total rate decreases. Our problem formulation assumes $\alpha=1 / 2$ for heuristics testing purpose.

\section{F. Multicommodity flow problem and its heuristics}

Our problem belongs to a class of more general flow problems, namely the multicommodity flow problem (MFP) (Leighton et al. [12]). However, our formulation is single-path, as opposed to the multi-path formulation in MFP. Additionally, we do not assume a fixed demand for each source-destination pair, but express such a demand with utility function. MFP heuristics may be found in many works e.g. Garg and Konemann [7], Fleisher [6].

\section{G. Heuristics for NUM with single-path routing}

Drwal [4] defines Utility-Maximizing Network Design problem understood as joint optimization over rates and paths (single-path). The work introduces Maximum Spanning Tree based algorithm, which gives a solution not less than a factor $O\left(\frac{1}{m}\right)$ of an optimal solution, where $m$ is the number of pairs.

Wang et al. [18] introduce several upper-bounds to estimate the performance gap between multi- and single-path solutions of joint optimization of transmission rates and paths. They introduce two heuristics: vertex-projection and greedy-branchand-bound.

\section{MAIN RESULTS}

In our paper we compared experimentally several heuristics to the NUM problem: MstNum (see alg 1), IterativeMstNum (see alg 2), LPRoundingNum (see alg 3), LPBestPathNum (see alg 4) and IterativeLPBestPathNum (see alg 5).

Our main contribution is the design of two heuristics: IterativeMstNum (an iterative procedure based on maximum spanning tree) and IterativeLPBestPathNum (an iterative procedure based on LP solver).

The other heuristics may be found in literature: MstNum [4], LPRoundingNum [4] [18] and LPBestPathNum [18].
All the algorithms are implemented and compared experimentally with MilpNum (see alg 6), which gives an optimal solution.

\section{PROBLEM DEFINITION}

Let's assume:

1) $G=(V, E)$ an undirected graph representing a network, where $V$ is a set of nodes and $E$ a set of edges (this model could also be defined for a directed graph)

2) $c: E \rightarrow[0, \infty)$ capacity function

3) $S$ a set of users

4) $F_{s t}=\left\{\left(s_{k}, t_{k}\right) \in V \times V: k \in S\right.$, and $\left.s_{k}, t_{k} \in V\right\}$ a set of (source,target) pairs - demands for transmission

5) $u: S \times[0, \infty) \rightarrow[0, \infty)$ utility function

6) $u(k, x)=u_{k}(x)$ utility value of transmission rate $x$ for the user $k \in S$

The problem is to find a set of pairs $\left\{\left(x_{k}, P_{k}\right): k \in S\right\}$, where $x_{k}$ is a non-negative transmission rate assigned to the user (2), $P_{k}$ is a transmission path in the graph $G$ from $s_{k}$ to $t_{k}$, assignments do not exceed (altogether) the edge capacity (3) and rates are maximized (1). Such an extended NUM problem is a generalization of the basic NUM formulated by Kelly [10].

$$
\max \sum_{k \in S} u_{k}\left(x_{k}\right)
$$

For the purpose of the article we assume $\forall_{i, j, \in S} \quad u_{i}=u_{j}$.

$$
\begin{gathered}
\forall_{k \in S} \quad x_{k} \geq 0 \\
\forall\{u, v\} \in E(G) \quad \sum_{k, \text { s.t. }\{u, v\} \in E\left(P_{k}\right)} x_{k} \leq c(u, v)
\end{gathered}
$$

MILP formulation: In MILP each flow $f_{k}$ takes a single path $P_{k}$ from the source $s_{k}$ to the target $t_{k}$. We introduce a real variable $f_{k u v}$ which indicates the amount of flow $f_{k}$ passing via edge $(u, v)$. The variable $f_{k u v}$ is defined to indicate $u \rightarrow v$ flow direction and $f_{k v u}$ reverse. We rewrite the constraint (3) as (4). We set a requirement that the flow is balanced (5), except source and terminal nodes (6)(7).

$$
\forall_{\{u, v\} \in E(G)} \sum_{k, \text { s.t. }\{u, v\} \in E\left(P_{k}\right)} f_{k u v}+f_{k v u} \leq c(\{u, v\})
$$

$$
\forall_{k \in S} \forall_{u \in V \backslash\left\{s_{k}, t_{k}\right\}} \sum_{v:\{v, u\} \in E(G)} f_{k v u}=\sum_{w:\{u, w\} \in E(G)} f_{k u w}
$$

$$
\begin{aligned}
& \forall_{k \in S} \sum_{w:\left\{s_{k}, w\right\} \in E(G)} f_{k s_{k} w}=x_{k} \\
& \forall_{k \in S} \sum_{v:\left\{v, t_{k}\right\} \in E(G)} f_{k v t_{k}}=x_{k}
\end{aligned}
$$

We introduce a binary variable $y_{k u v}$ which indicates whether flow $f_{k}$ is passing via edge $(u, v)$ (8). The variable 
$y_{k u v}$ is defined to indicate $u \rightarrow v$ flow direction and $y_{k v u}$ reverse.

$$
\forall_{k \in S} \forall_{\{u, v\} \in E(G)} y_{k u v}, y_{k v u} \in\{0,1\}
$$

We bind the variable $y_{k u v}$ with the variable $f_{k u v}$ using two additional constraints (9)(10).

$$
\begin{gathered}
\forall_{k u v: k \in S,\{u, v\} \in E(G)} \quad f_{k u v} \leq y_{k u v} \cdot c(\{u, v\}) \\
\forall_{k u v: k \in S,\{u, v\} \in E(G)} y_{k u v} \leq f_{k u v} \cdot M
\end{gathered}
$$

where $M$ is a large number.

In practice, fair allocations of capacity are needed. This can be obtained by choosing the utility function properly. In literature [3] [10] [4] most common is a family of functions:

$$
u_{k}\left(x_{k}\right)= \begin{cases}w_{k} \frac{1}{1-\alpha} x_{k}^{1-\alpha}, & \alpha>0, \alpha \neq 1 \\ w_{k} \log \left(x_{k}\right), & \alpha=1\end{cases}
$$

For the experiment purpose, we have chosen $\alpha=1 / 2$, which gives the function (12). We assume flow value to be non-negative (2).

$$
\forall k \in S \quad u_{k}(x)=u(x)=2 \sqrt{x}
$$

We approximate the function (12) with three linear functions over range $x \in[0,1]$ (see fig 1 ). Such a choice stems from our assumption $\forall\{u, v\} \in E(G) c(u, v)=1$. If links differ in terms of capacity, approximation function could range from 0 to max edge capacity. For the approximation purpose, an additional equation is introduced (14). The goal function (1) is rewritten as (13).

$$
\max \sum_{k \in S}\left(a_{1} \cdot t_{1, k}+a_{2} \cdot t_{2, k}+a_{3} \cdot t_{3, k}\right)
$$

where $a_{1}=12.00, a_{2}=3.00, a_{3}=1.3(3)$

$$
\forall_{k \in S} \quad x_{k}=t_{1, k}+t_{2, k}+t_{3, k}
$$

where $0 \leq t_{1, k} \leq 1 / 36,0 \leq t_{2, k} \leq 8 / 36$, and $0 \leq t_{3, k}$

Relaxed LP formulation: We relax the $y_{k u v}$ variable to allow the flow to split and flow via multiple paths (15). Such relaxation formulates the problem known in literature as MultiCommodity Flow (MCF). Since this is a linear program, MCF can be solved in polynomial time [15].

$$
\forall_{k \in S} \forall_{\{u, v\} \in E(G)} y_{k u v}, y_{k v u} \in[0,1]
$$

In this NUM formulation all constraints $\{(2),(4),(5),(6)$, (7), (14) $\}$ hold except $\{(8),(9),(10)\}$, which are replaced with (15).

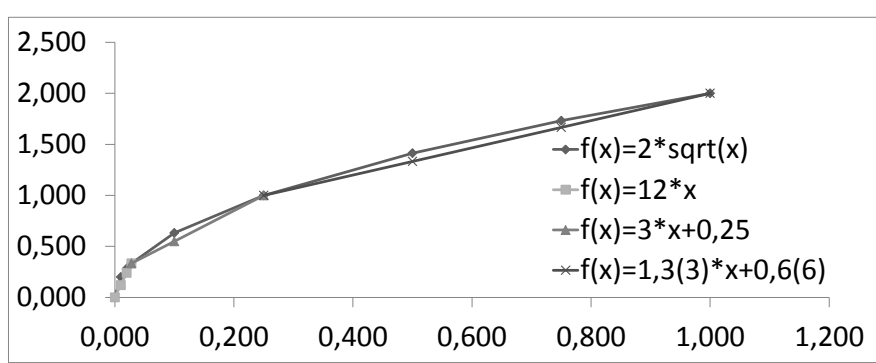

Fig. 1. Approximation of utility function $u_{k}(x)=2 \sqrt{x}$

\section{Algorithms}

To solve the NUM problem we have implemented the algorithms below. Some of them have been designed by us and some taken from literature (see chapter III Main results).

1) $\operatorname{MstNum}\left(G, c, F_{s t}\right)$ (see alg 1)

2) IterativeMstNum $\left(G, c, F_{s t}\right.$ ) (see alg 2)

3) LPRoundingNum $\left(G, c, F_{s t}\right.$ ) (see alg 3)

4) LPBestPathNum $\left(G, c, F_{\text {st }}\right.$ ) (see alg 4)

5) IterativeLPBestPathNum $\left(G, c, F_{\text {st }}\right.$ ) (see alg 5)

6) $\operatorname{MilpNum}\left(G, c, F_{s t}\right.$ ) (see alg 6)

All the algorithms assume the following input parameters:

- $G$ graph representing a network

- $c$ capacity function

- $F_{s t}$ a set of demands for transmission

and output parameters:

- $F A S$ such a set of pairs $\left\{\left(x_{k}, P_{k}\right): k \in S\right\}$ that constraints (2)(3) are satisfied

- $U$ aggregate utility function value.

\section{A. MST based NUM}

The algorithm MstNum is based on [4]. It uses single MST (Maximum Spanning Tree) run (line 1). All flow paths belong to the MST (line 3). If competing flows pass the same edge, the edge capacity is shared among the flows proportionally with the use of $\min$ function (line 11).

In the worst case (having a network with $|E|=m$ parallel edges spanned between two vertexes), assuming $\forall_{e \in E} c(e)=$ $k$, and $|S|=m$, the heuristics MstNum gives a ratio of a solution to an optimal solution equal to $1 / \mathrm{m}$. It is shown in [4] that the algorithm never returns a solution that is less than a factor $O\left(\frac{1}{m}\right)$ of an optimal solution.

\section{B. Iterative MST based NUM}

The algorithm IterativeMstNum offers a large improvement over MstNum with respect to maximization of the goal function. It consists of two phases (init phase and iterative phase). The first phase (init) runs MstNum (line 1). The second phase runs an iterative utility improvement procedure (lines 3-28). Within each iteration (a single while loop), $|S|$ solutions are found (line 4-21), compared and the best one is selected (line 22) to produce the input flow allocation set (FAS) for the next iteration (line 26). To find an $i$-th solution (within an iteration) the algorithm produces $i$-th MST (line 16) taking $c^{\prime}$ as the 

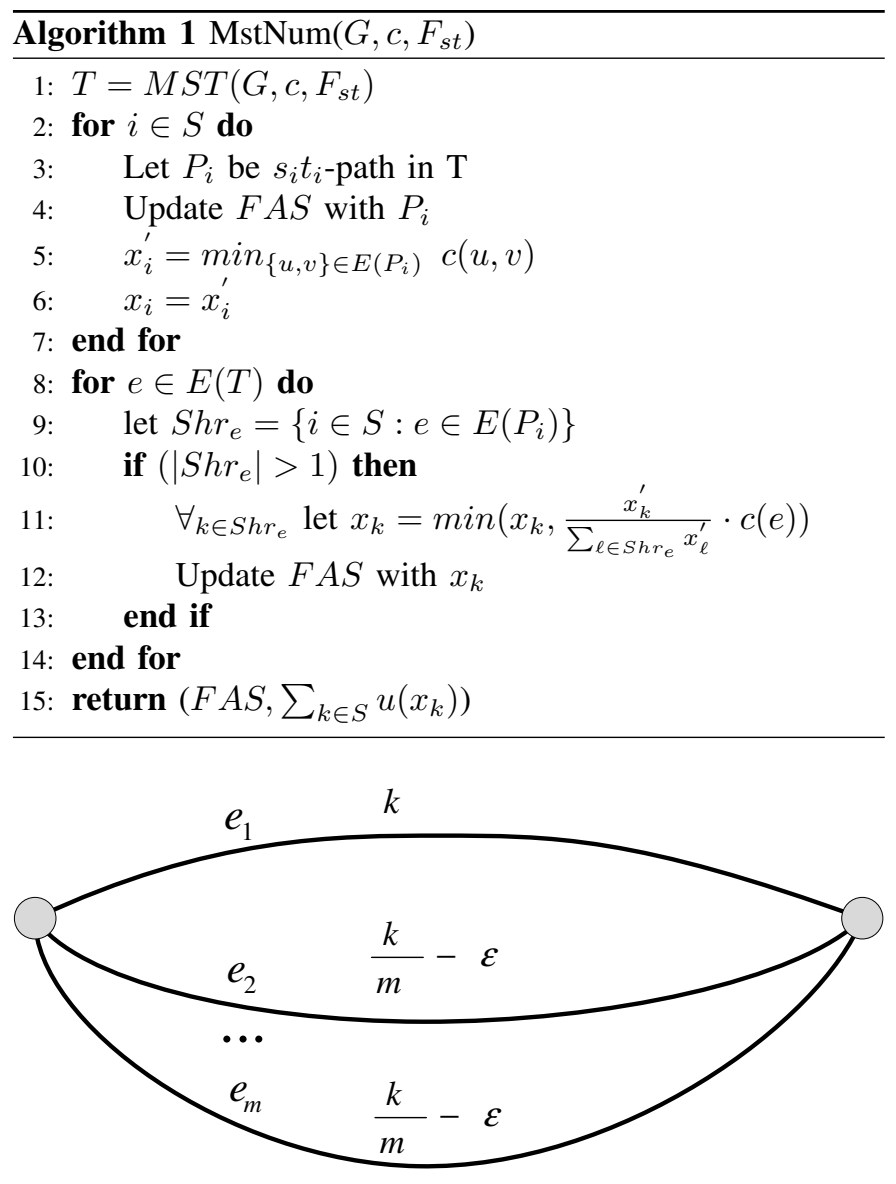

Fig. 2. Worst case for IterativeMstNum

graph edge capacity (lines 6-15), which equals $c$ decreased by the capacity used by the other users, and re-routes $i$-th flow to a possibly better location within the $i$-th MST (the new route may be the same as the previous one) (line 17). The $\Delta_{i}$ is computed as the aggregated sum of $i$-th flow utility difference and all $j$-th flow utility difference (line 20). The best solution is selected $\Delta_{\max }$ (line 22). If the objective improvement is less or equal to $\Delta_{\varepsilon}$, the stop condition is satisfied (line 24).

In the worst case (see fig 2), assuming $|S|=m$, a ratio of a solution to an optimal solution is determined by the equation (16). For $m \gg 1$ the ratio approximates to $1 / 2$. The algorithm IterativeMstNum never returns a solution that is less than a factor $O\left(\frac{1}{2}\right)$ of an optimal solution.

$$
\frac{s o l}{o p t}=\frac{1}{2-1 / m}
$$

However, for practical networks the heuristics exposes a solid ratio (see chapter VI Computational results).

\section{Linear program relaxation and rounding NUM}

The algorithm LPRoundingNum (a slight improvement of the one given in [4]) begins with solving linear program relaxation in phase1 (line 3). Subsequently in phase2 (lines 514) a rounding is done by random selection of the single path for each user. Finally, in phase3 (line 16) the LP problem is

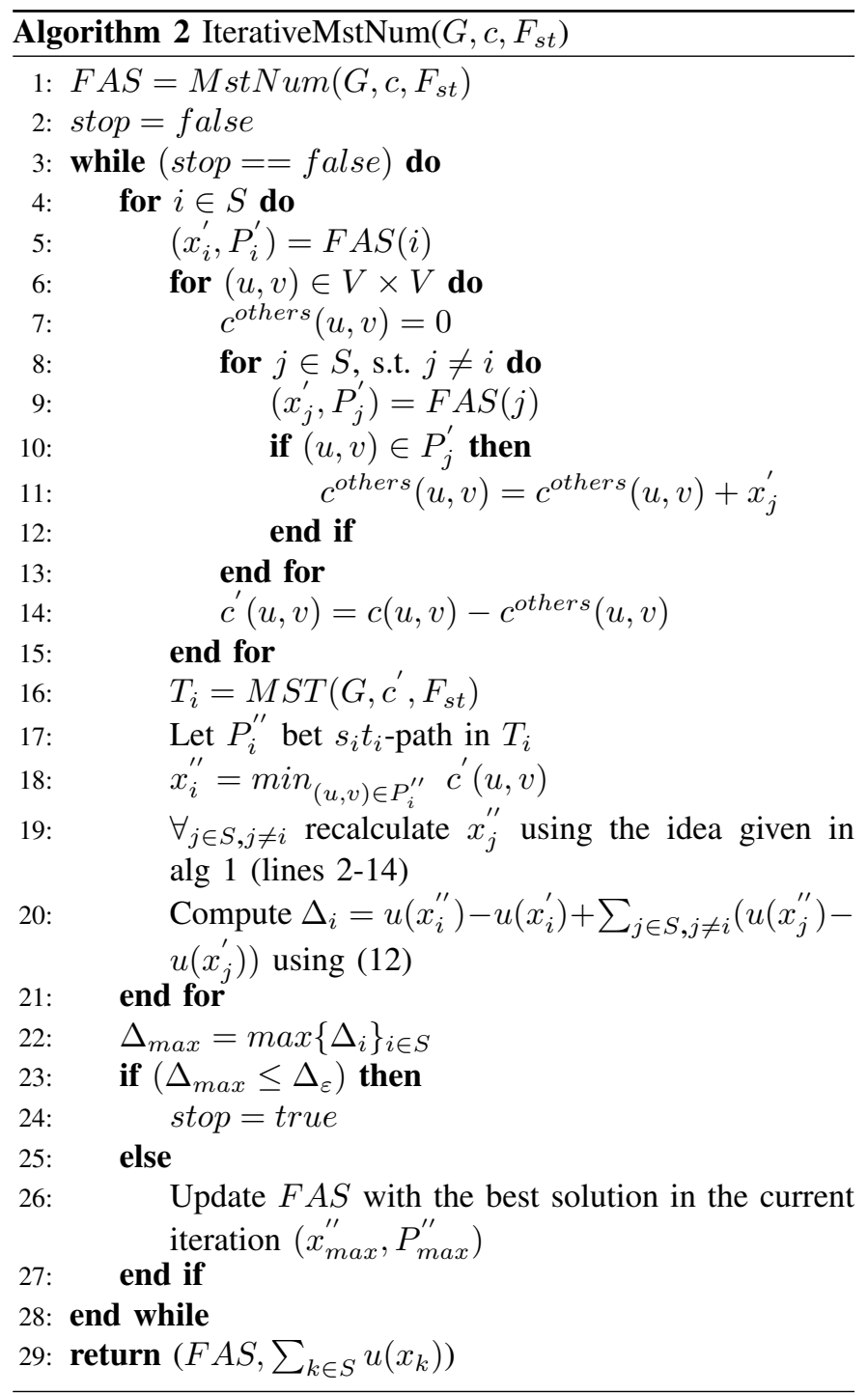

solved again taking fixed single paths identified in the previous phase as a constraint.

\section{LP Best Path NUM}

The algorithm LPBestPathNum begins with solving linear program relaxation (line 3 ). Within the relaxed LP solution, for each $k \in S$ the single max path (max min $y_{k u v}$ on the path) is identified as $P_{k}$ (line 6). The procedure is using the BFS (Breadth First Search) for that purpose. Finally, the LP problem is solved again taking fixed single paths identified (line 9) as a constraint. The algorithm is similar to the vertex projection method given in [18].

\section{E. Iterative LP Best Path NUM}

The algorithm IterativeLPBestPathNum constitutes $|S|+1$ iterations. In each $\{1, . .,|S|\}$ iteration, linear program relaxation is solved (line 7). Within the relaxed LP solution, for each $i \in S \backslash S^{\prime}$ the single max path $P_{i}\left(\max \min y_{i u v}\right.$ on the path) is selected (line 10). Among the $\left|S \backslash S^{\prime}\right|$-paths, the best $(\max ) P_{i}$ is selected and added to the model (line 14) as 

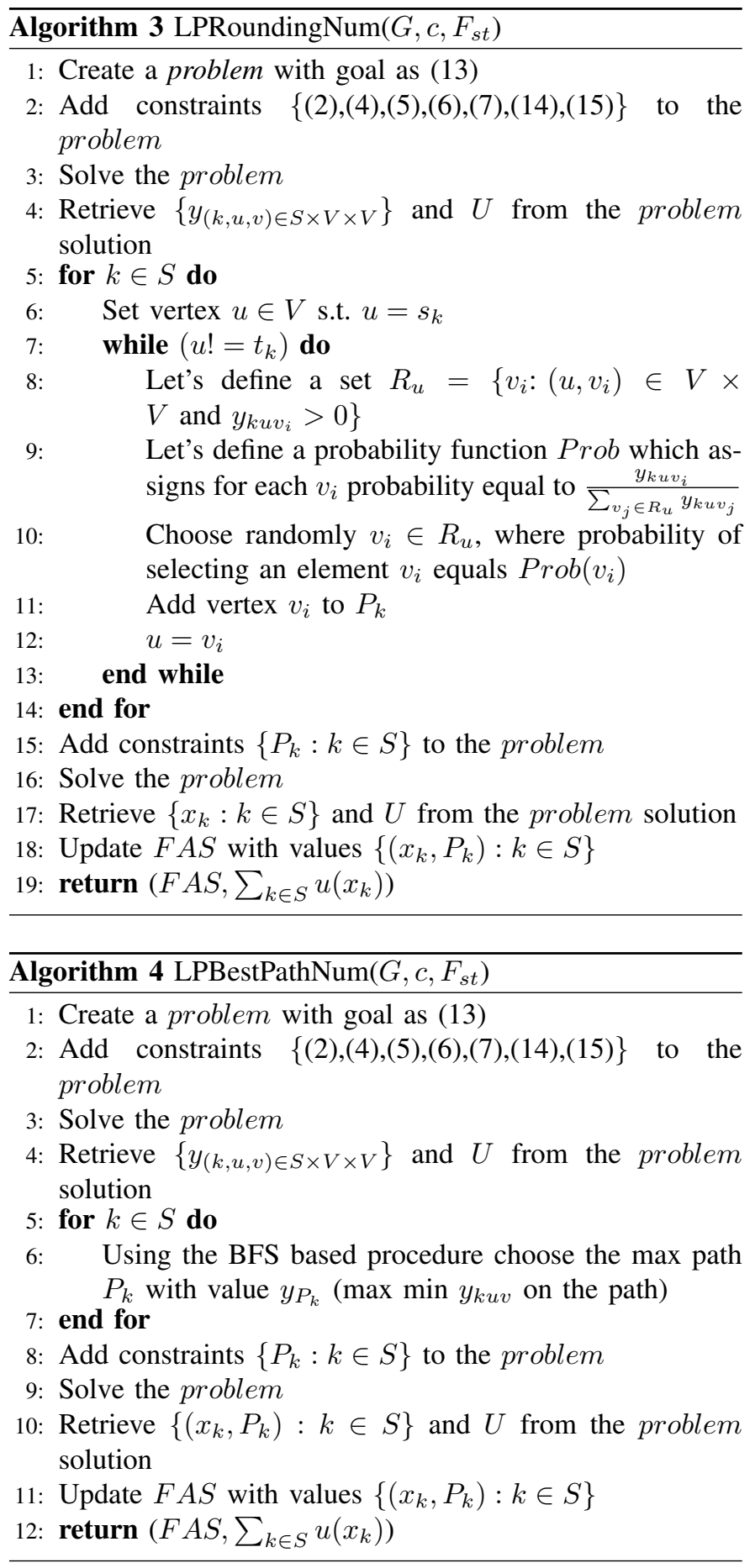

a constraint (random selection, since there may be more than one best path (line 13)). The constraint forces $i$-flow to use only $P_{i}$ edges in the next iterations.

In the last $|S|+1$ iteration the LP relaxation is solved assuming single fixed paths for all the $k \in S$ flows (line 17).

\section{F. Mixed integer linear program NUM}

The algorithm MilpNum is based on MILP formulation of the problem. It is a single phase algorithm (line 3 ).
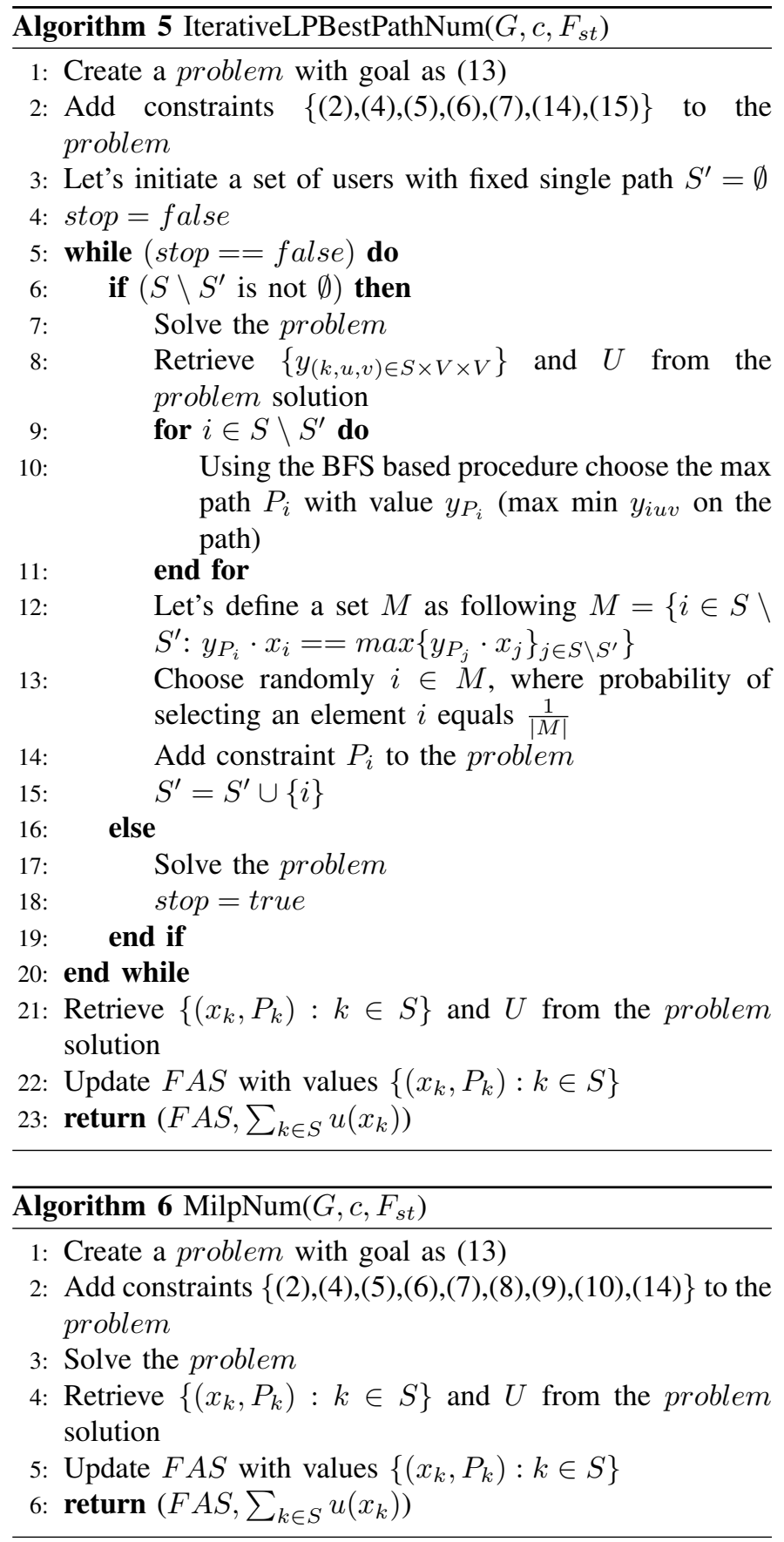

\section{COMPUTATIONAL RESUlts}

\section{A. Experiment Setup}

The algorithms were programmed in Python 2.7 with the use of CPLEX Python library 12.8.0. The algorithm MilpNum uses CPLEX executable. All the simulations were run on a PC with 2-core $2.7 \mathrm{GHz}$ and $8 \mathrm{~GB}$ RAM.

The experiments were conducted on five types of networks. The first network Net7 with 7 nodes and 10 links [9]. The second network Net22 with 22 nodes and 40 links [2]. In the second network nodes 1-8 and $\mathrm{T}$ (which is a network exit node) were source-destination nodes, whereas nodes 9 


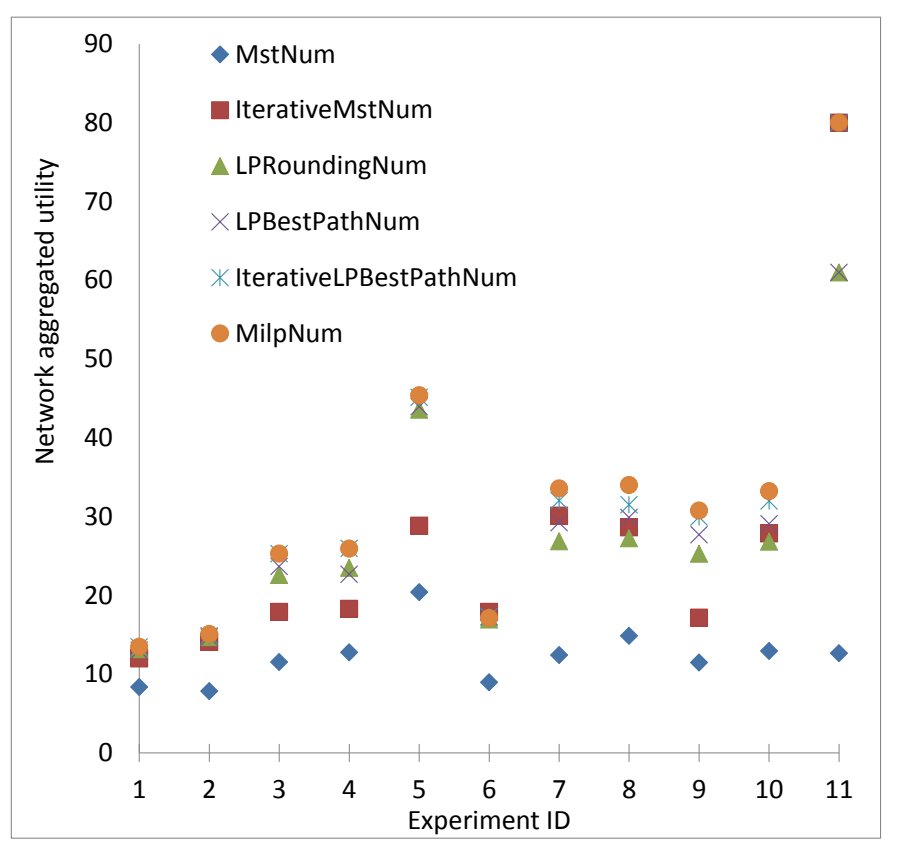

Fig. 3. Network aggregate utility (experiments $S 1-S 11$ )

21 were transit nodes. The third, grid network Net64 with $8 \times 8$ nodes. The fourth network Net42 with 40 disjoint paths spanned between node 1 and 42. Finally, the fifth, grid network Net400 with 20x20 nodes. In all the networks above each link had a capacity equal to 1 .

1) Verification of algorithm performance for different network structure and size: The experiment parameters were as follows: S1 - Net7, 10 pairs as given in [9]; S2 - Net22, 10 users randomly selected $^{1}$; S3/4 - Net22, 20 users randomly selected ${ }^{1}$, S5 - Net22, 50 users randomly selected ${ }^{1}$; S6 Net64, 20 users: $10 \times(1,64)$ and $10 \times(8,57)$; S7/8 - Net64, 20 users randomly selected; S9/10 - Net64, 20 users randomly selected $^{2}$; S11 - Net42, 20 users $(1,42)$

The algorithm IterativeMstNum was run with $\Delta_{\varepsilon}=0.1$.

In each experiment, the algorithms: LPRoundingNum, LPBestPathNum and IterativeLPBestPathNum were run 20 times and the average values of objective $U_{a v g}$ and execution time $T_{\text {avg }}$ are presented.

2) Verification of algorithm performance for different number of users: Additional 10 experiments (on Net64) were conducted to demonstrate an increase in utility and time upon an increase in the number of users: S12 - 10, S13 - 20, ... , S21 - 100 users ${ }^{2}$.

3) Verification of algorithm performance for a large network: Finally, additional 3 experiments were conducted to capture utility and time for the Net400: S22 - 20, S23 - 50, S24 - 100 users ${ }^{2}$.

${ }^{1} 50 \%$ of the pairs constitute internal traffic (between nodes $1-8$ ), and $50 \%$ of the pairs constitute external traffic (between nodes $1-8$ and $\mathrm{T}$ )

2 Traffic sources and targets were located on the grid edge, and were randomly selected. Each smaller user set was a subset of a larger set.

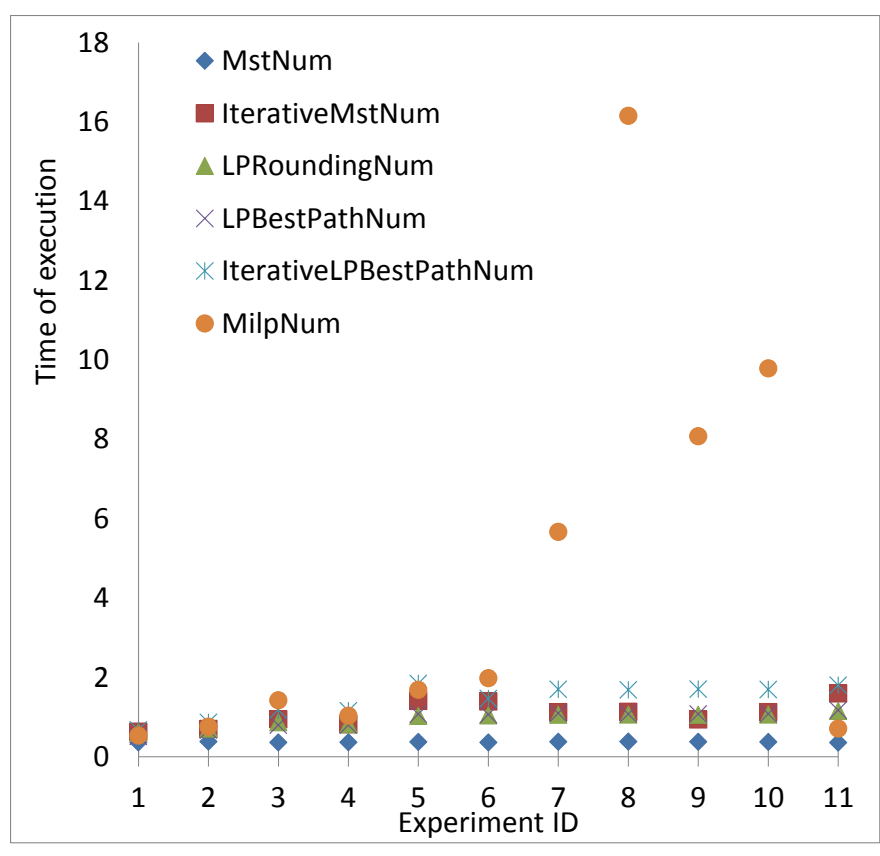

Fig. 4. Time of execution $\sqrt[4]{T}$ [sec.] (experiments $S 1-S 11$ )

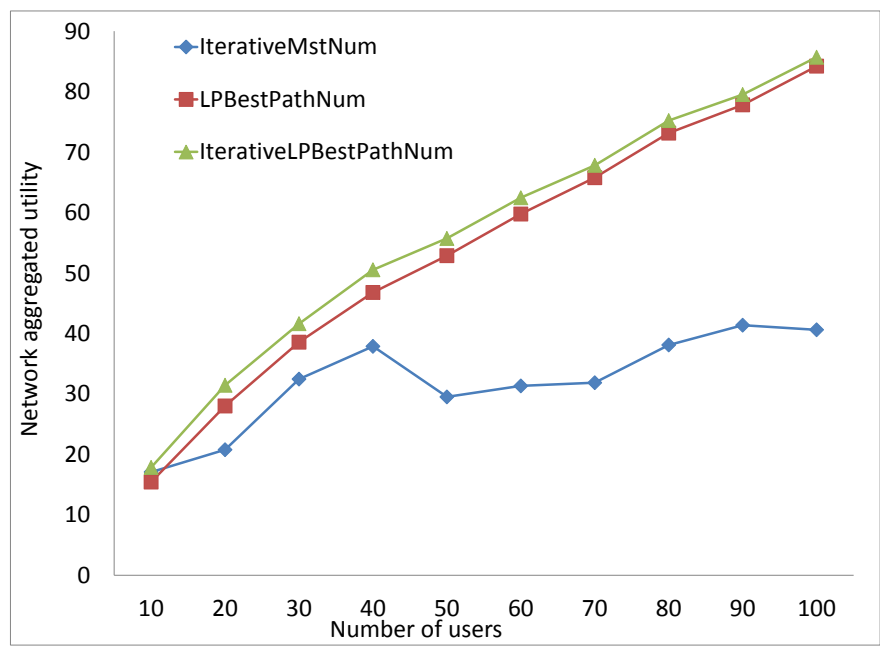

Fig. 5. Network aggregate utility in function of number of users (experiments $S 12-S 21)$

\section{B. Performance of IterativeMstNum heuristics}

1) Different network structure and size - experiments $S 1$ S11: IterativeMstNum vs MstNum. The results from $S 1-$ $S 11$ demonstrate a large improvement in the objective value of the algorithm IterativeMstNum in comparison to MstNum (Fig. 3) - efficiency (utility) was even several times higher as high.

IterativeMstNum vs LPRoundingNum. The utility values of IterativeMstNum observed in $S 1-5,9$ were not as high as the average ones achieved by LPRoundingNum (utility of LPRoundingNum was higher by $4 \%-51 \%$ (Fig. 3)). On the other hand, in $S 6-8, S 10-11$ the results were in favor of IterativeMstNum (utility of IterativeMstNum was higher by 


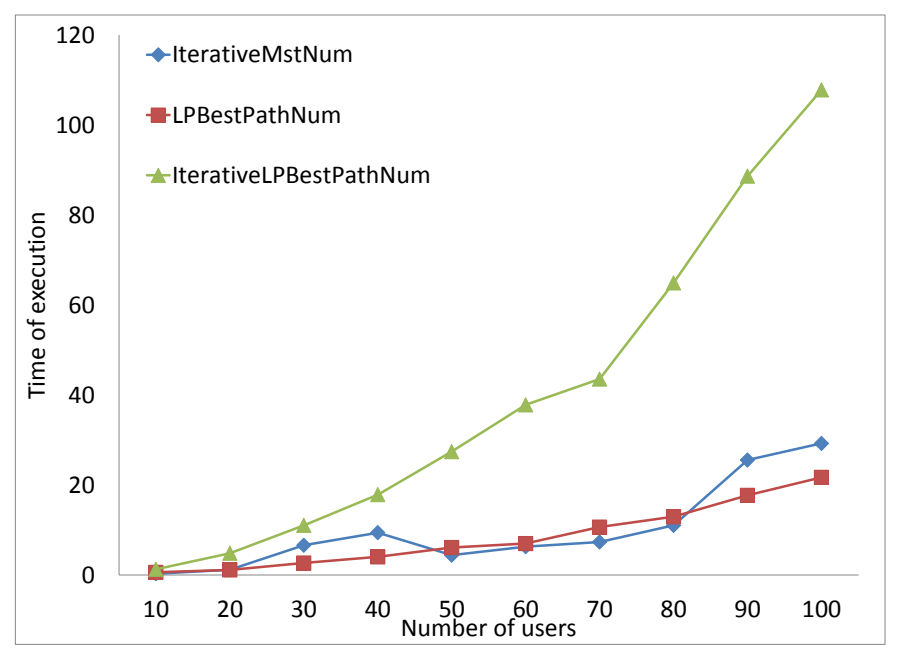

Fig. 6. Time of execution [sec.] in function of number of users (experiments $S 12-S 21)$

TABLE I

UTILITY AND TIME FOR A LARGE NETWORK (EXPERIMENTS $S 22-S 24$ )

\begin{tabular}{|c|c|c|c|c|c|c|c|}
\hline 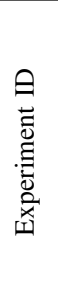 & 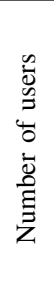 & 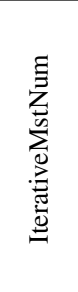 & & 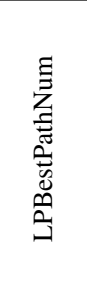 & & 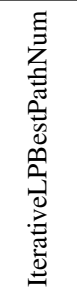 & \\
\hline & & $U$ & $T[s]$ & $U_{a v g}$ & $T_{a v g}[s]$ & $U_{a v g}$ & $T_{a v g}[s]$ \\
\hline S22 & 20 & 33,36 & 10,96 & 31,54 & 27,09 & 36,18 & 139,78 \\
\hline S23 & 50 & 38,85 & 30,34 & 63,59 & 341,51 & 77,46 & 6152,44 \\
\hline S24 & 100 & 36,49 & 208,56 & 109,40 & 5129,93 & - & - \\
\hline
\end{tabular}

\section{$4 \%-31 \%$ (Fig. 3))}

When it comes to the observed execution time, the algorithms IterativeMstNum and LPRoundingNum exposed similar performance, slightly in favour of LPRoundingNum (Fig. 4).

IterativeMstNum vs MilpNum. The objective values of IterativeMstNum observed in the experiments $S 6, S 11$ were very close to the ones achieved by MilpNum. In the experiment $S 6$ the value of MilpNum, lower than the value of IterativeMstNum, can be explained by an approximation error (Fig. 3). For a small number of users $(S 1, S 2)$ the observed distance from the optimum (MilpNum) was not more than $12 \%$, for a larger number of users $(S 3-S 5, S 7-10)$ it ranged between $11 \%$ and $80 \%$.

2) Different number of users - experiments $S 12-S 21$ : IterativeMstNum vs LPBestPathNum. In general, the observed utility of IterativeMstNum increased when the number of users grew, however the function was not monotonic. The utility was comparable with the one achieved by LPBestPathNum when the number of users was not high, up to 40 users for Net64 (Fig. 5). Then, the utility difference was not higher than $35 \%$. When the number of users was 10 , for Net64, the utility of IterativeMstNum was higher by $11 \%$. This number of users was correlated with the number of user-disjoint paths.

The time of execution of IterativeMstNum was comparable with the one achieved by LPBestPathNum regardless of the number of users (Fig. 6).

3) Large network - experiments $S 22-S 24$ : IterativeMstNum vs LPBestPathNum. The utility of IterativeMstNum was comparable with the one achieved by LPBestPathNum when the number of users was not high (TABLE I). When number of users was 20, for Net400, the utility of IterativeMstNum was higher by $5.8 \%$. The same remark on user-disjoint paths applies, as above.

The time of execution of IterativeMstNum was much smaller than the one achieved by LPBestPathNum (an order of magnitude when the number of users was high - TABLE I).

\section{Performance of LPBestPathNum and IterativeLPBestPath-} Num heuristics

1) Different network structure and size - experiments $S 1$ S11: LPBestPathNum vs LPRoundingNum. The average objective values of LPBestPathNum observed in the most of the experiments $S 1-S 11$ were superior to LPRoundingNum by up to $9.7 \%$ (Fig. 3), except for $S 1, S 4-1 \%$ and $3.6 \%$ opposite difference, respectively. Both heuristics demonstrated very similar execution time (Fig. 4).

LPBestPathNum vs IterativeLPBestPathNum. In $S 1-S 11$ the average observed objective values of IterativeLPBestPathNum were higher than values of LPBestPathNum by up to $14.5 \%$, except for the parallel edge network (Net42) by $31 \%$ (Fig. 3). However, IterativeLPBestPathNum exposed higher execution time due to additional $|S|-1$ iterations (Fig. 4).

IterativeLPBestPathNum vs MilpNum. In $S 1-S 6, S 11$ the optimal objective values (MilpNum) were higher than IterativeLPBestPathNum solution by not more than $1.5 \%$. In $S 7, S 9-10$ - by not more than $4.8 \%$. In $S 8$ - by $7.9 \%$.

2) Different number of users - experiments $S 12-S 21$ : IterativeLPBestPathNum vs LPBestPathNum. In $S 12-S 21$ the average objective values of IterativeLPBestPathNum and LPBestPathNum grew when the number of users grew. The utilities of IterativeLPBestPathNum were higher than those of LPBestPathNum (Fig. 5). The observed difference became smaller when the number of user grew. As observed, from $16 \%$ for 10 users to $2 \%$ for 100 users.

However, IterativeLPBestPathNum required much longer computation times than LPBestPathNum, with an increasing number of users (Fig. 6).

3) Large network - experiments $S 22$ - S24: IterativeLPBestPathNum vs LPBestPathNum. The utility difference between IterativeLPBestPathNum and LPBestPathNum was more visible for large networks (20x20 nodes), as observed $14.7 \%$ (20 users) $-21.8 \%$ (50 users) in favor of the former (TABLE I). However, the computation time of IterativeLPBestPathNum for a large networks and large number of users may not be practically attainable (TABLE I).

\section{Performance of MilpNum solver}

The experiments $S 7-10$ showed that MilpNum may expose a very long computation time to achieve an optimal solution, 
in the range of hours (Fig. 4). A possible, way around for MilpNum would be to configure the automatic termination when reaching a defined solution gap. As an example, in the experiment S9 MilpNum gave the value 26,22 (gap 16,95\%) in $12 \mathrm{~s}$ and $29(5,75 \%)$ in 90s. IterativeLPBestPathNum gave 29,928 in 8,4 s. In $S 10$ MilpNum gave $30(11,11 \%)$ in 12 s and $31,66(5 \%)$ in $268 \mathrm{~s}$. IterativeLPBestPathNum gave 31,962 in $8,2 \mathrm{~s}$.

\section{CONCLUSIONS}

Since our problem is NP-hard, search for optimum (MilpNum) may not be practically attainable even for medium-sized grid networks ( $8 \times 8$ nodes) and a small number of users $(\sim 20)$.

The experiments show that the algorithm LPBestPathNum demonstrates a reasonably good trade-off between computing time and precision of the utility value.

As observed, our LP-based heuristics IterativeLPBestPathNum demonstrates up to $\sim 15 \%$ higher utility, on networks with up to 64 nodes and 50 users, but it is slower, in comparison to LPBestPathNum. The difference in utility may be a few percent bigger for large grid networks (20x20 nodes) and a high number of users $(\sim 50)$, and even more for a special type of network - parallel edges. The bigger difference is caused by the edge allocation conflict. The IterativeLPBestPathNum reduces the probability of such conflicts by iterative path fixing (a single path fixed for each iteration).

The utility values of IterativeLPBestPathNum, on network with up to 64 nodes and 50 users, are lower than MilpNum by only $\sim 2 \%$ on average.

The successful IterativeLPBestPathNum computations were conducted for grid networks with up to $20 \times 20$ nodes and 50 users. For comparison, the successful and not terminated MilpNum computations were conducted for grid networks with up to $8 \times 8$ nodes and 20 users.

As observed, our MST-based heuristics IterativeMstNum ensures reasonably good utility in certain cases. When the number of users is not high (the number is correlated with the number of user-disjoint paths), the IterativeMstNum utility is lower than MilpNum by not more than $\sim 10 \%$. With such an assumption it has efficiency comparable to LPBestPathNum. When the number of users grows, the gap between IterativeMstNum and LPBestPathNum increases. The IterativeMstNum has utility which is equal to optimal for a special type of network - parallel edge network, since it avoids allocating the same edge to competing streams if alternative ones are available. In such networks, it may be much more efficient than LPBestPathNum (e.g. S11-30\%).

On average, for the observed cases, the IterativeMstNum utility value is lower than MilpNum by $\sim 25 \%$.

Additionally, IterativeMstNum has computing time comparable to LPBestPathNum for small and medium-sized networks. For large networks and a high number of users, it may be an order of magnitude faster than LPBestPathNum. Finally, it does not use LP solver but a simple MST-based iterative procedure.

\section{REFERENCES}

[1] A. Amokrane, R. Langar, R. Boutaba, and G. Pujolle. Flow-based management for energy efficient campus networks. IEEE Transactions on Network and Service Management, 12(4):565-579, Dec 2015. 10.1109/TNSM.2015.2501398.

[2] A.P. Bianzino, L. Chiaraviglio, M. Mellia, and J.L. Rougier. Grida: Green distributed algorithm for energy-efficient ip backbone networks. Computer Networks, 56(14):3219-3232, September 2012. https://doi.org/10.1016/j.comnet.2012.06.011.

[3] M. Chiang, S. H. Low, A. R. Calderbank, and J. C. Doyle. Layering as optimization decomposition: A mathematical theory of network architectures. Proceedings of the IEEE, 95(1):255-312, Jan 2007. 10.1109/JPROC.2006.887322.

[4] M. Drwal. Approximation algorithms for utility-maximizing network design problem. In Henry Selvaraj et al., editor, Progress in Systems Engineering: Proceedings of the Twenty-Third International Conference on Systems Engineering, volume 366 of Advances in Intelligent Systems and Computing. Springer International Publishing, Cham, 2015. https://doi.org/10.1007/978-3-319-08422-0-60.

[5] S. Even, A. Itai, and A. Shamir. On the complexity of time table and multi-commodity flow problems. In IEEE, editor, 16th Annual Symposium on Foundations of Computer Science 13-15 October 1975, October 1975. 10.1109/SFCS.1975.21.

[6] L. K. Fleischer. Approximating fractional multicommodity flow independent of the number of commodities. In 40th Annual Symposium on Foundations of Computer Science (Cat. No.99CB37039), pages 24-31, Oct 1999. 10.1109/SFFCS.1999.814573.

[7] N. Garg and J. Konemann. Faster and simpler algorithms for multicommodity flow and other fractional packing problems. In Proceedings 39th Annual Symposium on Foundations of Computer Science (Cat. No.98CB36280), pages 300-309, Nov 1998. 10.1109/SFCS.1998.743463.

[8] X. Huang, T. Yuan, and M. Ma. Utility-optimized flow-level bandwidth allocation in hybrid sdns. IEEE Access, 6:20279-20290, 2018. 10.1109/ACCESS.2018.2820682.

[9] P. Jaskóła, P. Arabas, and A. Karbowski. Simultaneous routing and flow rate optimization in energy-aware computer networks. International Journal of Applied Mathematics and Computer Science, 26(1):231-243, 2016. 10.1515/amcs-2016-0016.

[10] F.P. Kelly, A.K. Maulloo, and D.K.H. Tan. Rate control for communication networks: shadow prices, proportional fairness and stability. Journal of the Operational Research Society, 49(3):237-252, March 1998. https://doi.org/10.1057/palgrave.jors.2600523.

[11] J.W. Lee, M. Chiang, and R. A. Calderbank. Jointly optimal congestion and contention control based on network utility maximization. IEEE Communications Letters, 10(3):216-218, March 2006. 10.1109/LCOMM.2006.1603389.

[12] T. Leighton and S. Rao. Multicommodity max-flow min-cut theorems and their use in designing approximation algorithms. Journal of the ACM, 46(6):787-832, November 1999.

[13] M. Macit, V. C. Gungor, and G. Tuna. Comparison of qos-aware singlepath vs. multi-path routing protocols for image transmission in wireless multimedia sensor networks. Ad Hoc Networks, 19:132 - 141, 2014. https://doi.org/10.1016/j.adhoc.2014.02.008.

[14] J. Mo and J. Walrand. Fair end-to-end window-based congestion control. IEEE/ACM Transactions on Networking, 8(5):556-567, Oct 2000. 10.1109/90.879343

[15] M. E. Pfetsch and Ch. Liebchen. Multicommodity flows and column generation. In Lecture Notes. Zuse Institute Berlin, 2006.

[16] P. R. Satav and P. M. Jawandhiya. Review on single-path multi-path routing protocol in manet: A study. In 2016 International Conference on Recent Advances and Innovations in Engineering (ICRAIE), pages 1-7, Dec 2016. 10.1109/ICRAIE.2016.7939514.

[17] J. Wang, L. Li, S.H. Low, and J.C. Doyle. Cross-layer optimization in tcp/ip networks. IEEE/ACM Transactions on Networking, 13(3):582 595, June 2005. 10.1109/TNET.2005.850219.

[18] M. Wang, C. W. Tan, W. Xu, and A. Tang. Cost of not splitting in routing: Characterization and estimation. IEEE/ACM Transactions on Networking, 19(6):1849-1859, Dec 2011. 10.1109/TNET.2011.2150761.

[19] W. Wang, M. Palaniswami, and S. H. Low. Applicationoriented flow control: Fundamentals, algorithms and fairness. IEEE/ACM Transactions on Networking, 14(6):1282-1291, Dec 2006. 10.1109/TNET.2006.886318. 